\title{
Effects of Learning Parameters on Learning Procedure and Performance of a BPNN
}

\author{
hengchang Dai ${ }^{1,2}$ and Colin MacBeth' \\ 'British Geological Survey, and ${ }^{2}$ Department of Geology and Geophysics, Edinburgh University \\ (Received 29 April 1996; accepted 14 November 1996)
}

\begin{abstract}
We examined the effects of changing learning parameters on the learning procedure and performance of back-propagation neural networks used to pick seismic arrivals. The results show that such change mainly affects the speed of convergence of the learning procedures, and does not affect the BPNN structure and its overall performance.A relationship between the learning parameters and iteration number is obtained. This relationship may be used as a guide to check the convergence of the learning procedure and the BPNN performance. We also use a weight map of BPNN structure to analyze its interior and performance. Two BPNNs used to pick seismic arrivals from three-component and single-component seismograms have similar weight patterns and operate in a similar way, although they have different structures and trained by different training dataset. (C) 1997 Elsevier Science Ltd.
\end{abstract}

Keywords-Back-propagation neural network, Seismic arrivals picking, Pattern recognition, Learning procedure, Convergence.

\section{INTRODUCTION}

The back-propagation neural network (BPNN) trained by the generalized delta rule (Rumelhart, Hinton \& Williams, 1986) is successfully used in many fields, especially for pattern recognition, due to its learning ability. However, one of its major disadvantages is that it looks like a "black-box" and provides no insight into the physics of its processing (McCormack, 1991). As a result, it is difficult to understand how it processes. Its performance is affected by many factors, such as the network structure (the numbers of input nodes, output nodes, hidden layers, and hidden nodes), and training parameters (the learning rate, momentum rate, and thresholds of system and pattern errors) which form components of the generalized delta rule. The range of choice of these factors results in a variable performance and, consequently, makes comparisons difficult. It is unclear if and when the optimum solution has been reached.

Acknowledgements: This research was sponsored by the Global Seismology Research Group (GSRG) at the British Geological Survey (BGS), Edinburgh, and is published with the approval of the Director of BGS. The authors would like to thank anonymous reviewers for their comments and suggestions.

Requests for reprints should be sent to Hengchang Dai or Colin McBeth, British Geological Survey, Murchison House, West Mains Road, Edinburgh EH9 3LA, UK. Tel: +44 131-667-1000, Fax: +44 131-667-1877, E-mail: e_hcd@va.nmh.ac.uk (for Dr Hengchang Dai), e_gs33@va.nmh.ac.uk (for Dr Colin MacBeth).
Although some efforts have been devoted to selecting optimal values (Gorman and Sejnowski, 1988; Dowla Taylor \& Anderson, 1990; Fausett, 1994), such values can only be obtained by a process of trial and error which is often time-consuming and inconsistent. It makes the application of BPNNs difficult. There is a need to find an appropriate method which can efficiently select these factors and understand the BPNN performance. The most important selection of the training parameters of generalized delta rule are the learning rate $\eta$ and momentum rate $\alpha$. They may have different values depending upon the problems and the researcher's favourite choice. Usually, determination is arbitrary and their optimum values can only be obtained by a process of trial and error.

In order to investigate this effect, two questions should be answered: (1) how do they affect the training procedure? and (2) how do they affect the BPNN structure? The training procedure can be measured by using the iteration number for convergence. The trained BPNN structure can be measured by its weight pattern in a weight map which displays the distribution of weight values (Dai and MacBeth, 1994a). In this paper, we will investigate this effect by analyzing a BPNN specially applied to the problem of picking seismic arrivals from local earthquake data. We will introduce the approach for picking seismic arrivals, and analyze the effects of changing the learning parameters. 


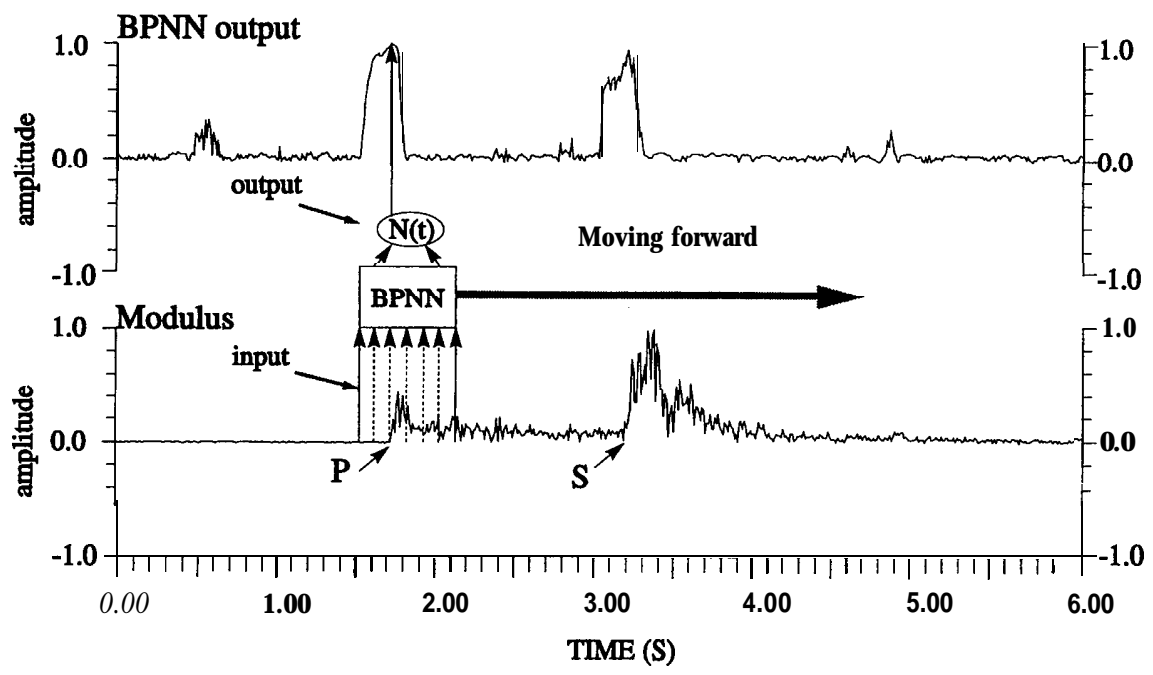

FIGURE 1. Schematic diagram showing the method of using a BPNN to pick seismic arrivals. Here, the input of the BPNN is a segment of the modulus of three-component recordings (the low diagram). A trained BPNN is treated as a filter in a sliding window across though the entire modulus trace. The output of the BPNN (the upper diagram) yields a time series which enhances the changes in the modulus to indicate the arrival onset. $P$ and $S$ indicate the $P$ - and $S$-arrivals.

\section{SEISMIC ARRIVAL PICKING}

Picking seismic arrival from background noise is a typical pattern recognition problem. In order to detect and locate a seismic event, the primary $(P)$ and secondary $(S)$ wave arrivals must be picked and their onset times must be measured. We have developed a BPNN approach to tackle this (Dai and MacBeth, 1995). This approach includes two phases: the training (learning) phase and the testing (generating) phase. In the training phase, some training dataset should be selected by an analyst using his experience and then be used to train the BPNN. After training, the BPNN is applied to the entire seismogram as a filter by using a sliding window along the entire modulus trace of three-component seismograms (Figure 1). The input of the BPNN is a segment of the modulus. The output of the BPNN then yields a time series which is interrogated for a decision regarding the seismic arrivals.

In such an application, the first task is to select a suitable BPNN structure, then a suitable training dataset, and also it is necessary to select suitable training parameters. In our early paper (Dai and MacBeth, 1995), we examined some factors which affect the performance. We found that: (1) a three-layer BPNN (with a single hidden layer) is sufficient; (2) there exist optimal numbers of both input nodes and hidden nodes and the input node number seems to have a large effect; (3) the performance is strongly dependent on the training dataset. A small training dataset may result in a poorly trained BPNN, and a large one may result in the learning procedure becoming too long. It seems better to train a BPNN with a small training dataset first, and then to improve its performance by subsequently adding more training dataset. Finally, by using a process of trial and error, we selected a three-layer BPNN which had an input layer, a hidden layer and an output layer (Figure 2). The input layer has 30 nodes corresponding to the segment of the input characteristic function which is chosen to include several complete cycles of a wave in the sliding window, the hidden layer has 10 nodes, and the output layer has two nodes flagging the result, $(0,1)$ for an arrival and $(1,0)$ for pure background noise.

In this approach, the BPNN is applied to pick as many changes from the background noise as possible and discard those which are neither $P$ - nor $S$-arrivals, so the training data should include the seismic arrival signal and background noise signal. Because both $P$ and $S$-arrivals appear to exhibit similar modulus characteristics, the training dataset only includes the

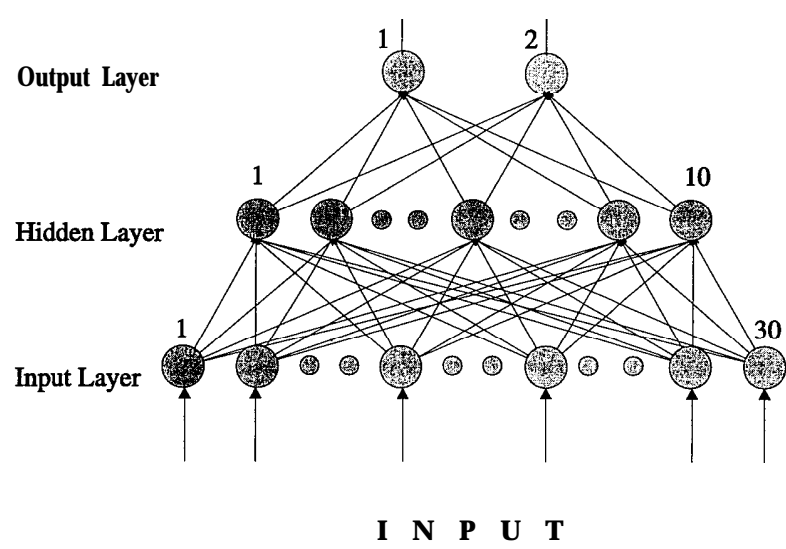

FIGURE 2. The structure of a BPNN for seismic arrival picking. It has three layers including an input layer with $\mathbf{3 0}$ nodes, a hidden layer with 10 nodes and an output layer with two nodes. The BPNN input is a segment of the modulus of three component seismograms. The two BPNN outputs indicate the input segment with $(0,1)$ as a P-arrival and $(1,0)$ as noise. 

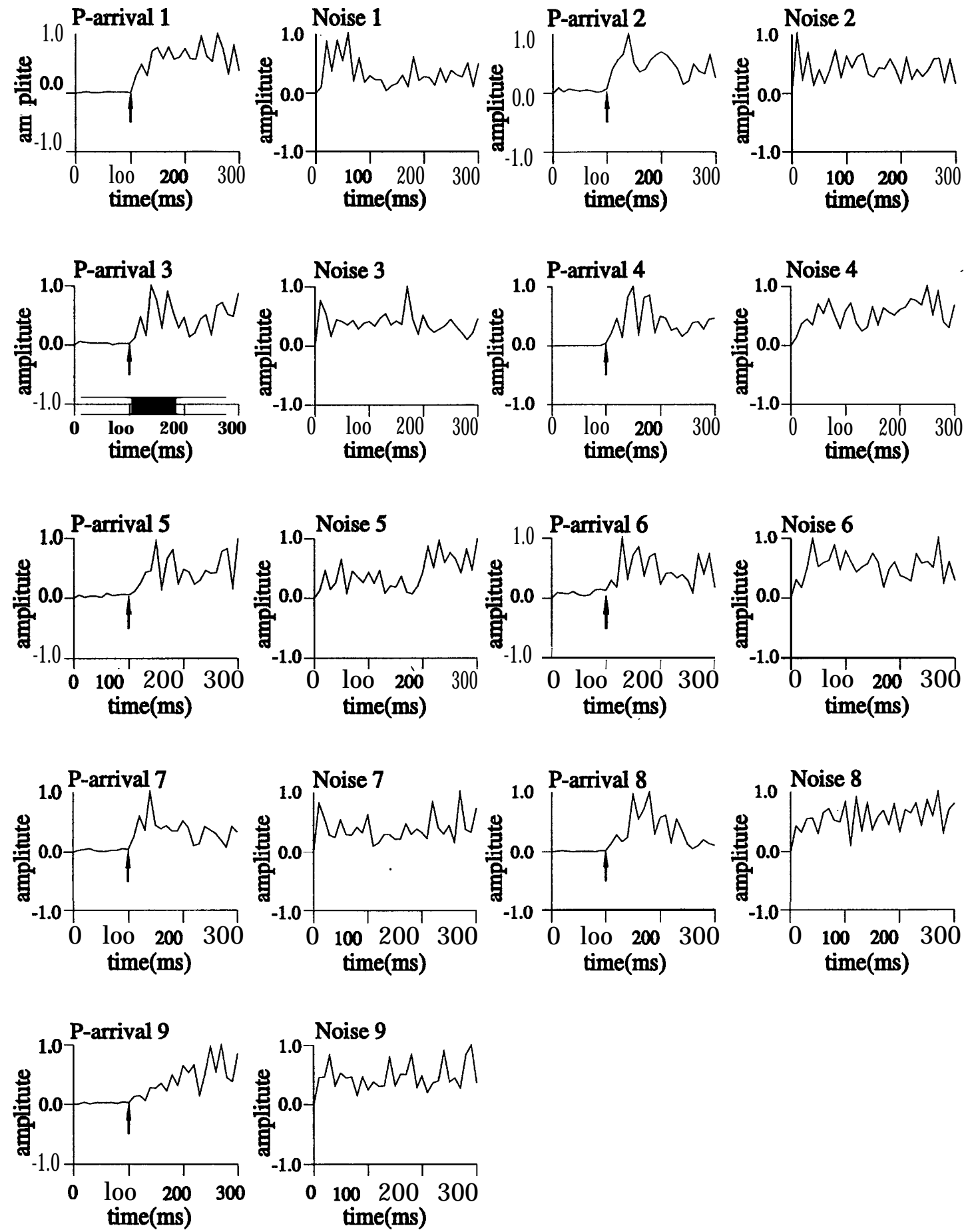

FIGURE 3. Nine P-arrival and nine background noise segments of the modulus of three-component recordings used for training a BPNN. Noise segments are extracted prior to the P-arrivals in the same seismograms. Arrows on P-arrival segments indicate arrival onset times used to train the BPNN, all at the eleventh sample. These segments are individually normalized before being input into the BPNN.

modulus segment of the $P$-arrivals and background noise which was extracted prior to the $P$-arrivals in the same seismograms. These segments are arranged so that the predicted onset time of every $P$-arrival lies at the eleventh sample. Finally nine pairs of $P$-arrivals and background noise segments (Figure 3) are selected for training in the study. With the above
BPNN structure and training dataset, this approach is applied to deal with several hundreds of three-component seismograms from a local earthquake network. It can detect $94 \% P$-arrivals and $90 \% S$-arrivals. $75 \%$ of the $P$-arrivals and $67 \%$ of the $S$-arrivals are estimated from their onset times within one sample increment (10 ms). 


\section{LEARNING PARAMETERS AND LEARNING CONVERGENCE}

The generalized delta rule which is used to train BPNNs can be mathematically written as:

$$
\Delta w_{i j k}(n+1)=\eta \delta_{i k} o_{i j}+\alpha \Delta w_{i j k}(n)
$$

where $\eta$ is the learning rate and $\alpha$ the momentum rate. $\Delta w_{i j k}$ is the change of the weighted connection between $n_{i j}\left(n_{i j}\right.$ is defined as the $j$ th node in the $i$ th layer) and $n_{i+1}$, $o_{i j}$ the output of $n_{i j}$, and $\delta_{i k}$ the change in error as a function of the change in the network input to the $n_{i+1} k$. The quantity $(n+1)$ indicates the $(n+1)$ th step. This equation means the change of weights at the $(n+1)$ th step should be similar to the change of weights undertaken at the $n$th step.
To train a BPNN, the first input pattern is presented to an initially randomized BPNN, and the weights (including thresholds of nodes) adjusted in all the connections. Other patterns are then presented in succession, and the weights adjusted from the previously determined values. This process continues until all patterns in the training set are exhausted (an iteration). The final solution is generally independent of the order in which the example patterns are presented. However, a final check can be performed by looking at the pattern error, $E_{\mathrm{p}}$, the sum of squares of the difference between desired output and BPNN output for each pattern, and system error, $E_{\mathrm{s}}$, the average of all pattern errors, to determine whether the final BPNN solution satisfies all of the patterns presented to it within a certain error.

TABLE 1

\begin{tabular}{|c|c|c|c|c|c|c|c|c|c|c|c|c|}
\hline & \multirow{2}{*}{$\begin{array}{l}\text { Iteration } \\
\text { number }\end{array}$} & \multicolumn{11}{|c|}{$\alpha$} \\
\hline & & 0.0 & 0.1 & 0.2 & 0.3 & 0.4 & 0.5 & 0.6 & 0.7 & 0.8 & 0.9 & 1.0 \\
\hline & \multirow[t]{3}{*}{0.1} & 7248 & 6523 & 5799 & 5074 & 4350 & 3625 & 2900 & 2175 & 1451 & 730 & NC \\
\hline & & 2.69 & 2.69 & 2.69 & 2.70 & 2.70 & 2.70 & 2.70 & 2.70 & 2.70 & 2.69 & \\
\hline & & 7248 & 6523 & 5798 & 5074 & 4349 & 3624 & 2899 & 2174 & 1450 & 725 & \\
\hline & \multirow[t]{3}{*}{0.2} & 3634 & 3274 & 2910 & 2547 & 2183 & 1819 & 1455 & 1091 & 728 & 368 & NC \\
\hline & & 2.70 & 2.69 & 2.70 & 2.70 & 2.71 & 2.71 & 2.72 & 2.72 & 2.72 & 2.71 & \\
\hline & & 3624 & 3262 & 2899 & 2537 & 2175 & 1812 & 1449 & 1087 & 725 & 363 & \\
\hline & \multirow[t]{3}{*}{0.3} & 2435 & 2191 & 1948 & 1705 & 1462 & 1218 & 974 & 729 & 486 & 247 & NC \\
\hline & & 2.70 & 2.70 & 2.71 & 2.71 & 2.72 & 2.73 & 2.74 & 2.75 & 2.74 & 2.75 & \\
\hline & & 2416 & 2174 & 1932 & 1691 & 1450 & 1208 & 966 & 725 & 483 & 242 & \\
\hline & \multirow[t]{3}{*}{0.4} & 1834 & 1651 & 1468 & 1285 & 1102 & 918 & 734 & 550 & 366 & 188 & NC \\
\hline & & 2.70 & 2.71 & 2.71 & 2.72 & 2.73 & 2.74 & 2.74 & 2.76 & 2.77 & 2.81 & \\
\hline & & 1812 & 1631 & 1449 & 1269 & 1087 & 906 & 725 & 544 & 363 & 181 & \\
\hline & \multirow[t]{3}{*}{0.5} & 1474 & 1327 & 1180 & 1033 & 886 & 739 & 591 & 443 & 295 & 151 & NC \\
\hline & & 2.70 & 2.71 & 2.72 & 2.73 & 2.74 & 2.75 & 2.77 & 2.78 & 2.79 & 2.87 & \\
\hline & & 1450 & 1304 & 1159 & 1015 & 870 & 725 & 580 & 435 & 290 & 145 & \\
\hline \multirow[t]{18}{*}{$\eta$} & \multirow[t]{3}{*}{0.6} & 1234 & 1111 & 989 & 866 & 743 & 620 & 496 & 372 & 248 & 124 & $\mathrm{NC}$ \\
\hline & & 2.70 & 2.71 & 2.72 & 2.73 & 2.75 & 2.76 & 2.78 & 2.80 & 2.80 & 2.97 & \\
\hline & & 1208 & 1087 & 966 & 846 & 725 & 604 & 483 & 362 & 242 & 121 & \\
\hline & \multirow[t]{3}{*}{0.7} & 1063 & 957 & 852 & 746 & 641 & 535 & 428 & 321 & 215 & 102 & NC \\
\hline & & 2.70 & 2.71 & 2.72 & 2.74 & 2.75 & 2.77 & 2.79 & 2.82 & 2.81 & 3.19 & \\
\hline & & 1035 & 931 & 828 & 724 & 621 & 518 & 414 & 310 & 207 & 104 & \\
\hline & \multirow[t]{3}{*}{0.8} & 935 & 842 & 750 & 657 & 564 & 471 & 378 & 284 & 190 & 85 & NC \\
\hline & & 2.69 & 2.71 & 2.72 & 2.74 & 2.76 & 2.78 & 2.80 & 2.81 & 2.84 & 3.45 & \\
\hline & & 906 & 815 & 725 & 634 & 543 & 453 & 362 & 272 & 181 & 91 & \\
\hline & \multirow[t]{3}{*}{0.9} & 836 & 753 & 670 & 588 & 505 & 422 & 338 & 254 & 171 & 73 & NC \\
\hline & & 2.68 & 2.98 & 2.72 & 2.73 & 2.75 & 2.78 & 2.81 & 2.83 & 2.86 & 3.57 & \\
\hline & & 805 & 725 & 644 & 564 & 483 & 403 & 322 & 242 & 161 & 81 & \\
\hline & \multirow[t]{3}{*}{1.0} & 756 & 681 & 606 & 532 & 457 & 382 & 307 & 231 & 156 & 67 & NC \\
\hline & & 2.68 & 2.70 & 2.72 & 2.73 & 2.76 & 2.78 & 2.81 & 2.83 & 2.97 & 3.52 & \\
\hline & & 725 & 652 & 580 & 507 & 435 & 362 & 290 & 217 & 145 & 73 & \\
\hline & \multirow[t]{3}{*}{1.1} & 691 & 622 & 554 & 486 & 418 & 350 & 281 & 212 & 143 & 65 & NC \\
\hline & & 2.67 & 2.69 & 2.71 & 2.73 & 2.76 & 2.78 & 2.81 & 2.83 & 2.90 & 3.37 & \\
\hline & & 659 & 593 & 527 & 461 & 395 & 329 & 264 & 197 & 131 & 66 & \\
\hline
\end{tabular}

The iteration number for training with different learning rate $\eta$ ranged from 0.1 to 1.1 and momentum rate $\alpha$ ranged from 0.0 to 1.0 . In each cell, the top number is the number of training iteration, the middle one is the system error $\left(x_{10^{-5}}\right)$, and the bottom one is the predicted iteration by using eqn (2). NC means nonconvergence. This BPNN is trained with seven pairs of P-arrival and background noise segments. 
The convergence speed (iteration number) of the training procedure is obviously dependent on the learning rate $\eta$, and momentum rate $\alpha$. Naturally, the higher the learning rate is, the larger the change in the weights. For practical purposes, it should be chosen to be as high as possible. However, if the learning rate is too large, it will lead to unstable oscillation (Pao, 1988). This oscillation might be filtered out by the momentum term. However selecting them is somewhat difficult as their values depend on the nature of the problem. Here we attempt to obtain a relationship between the learning parameter and the iteration number by analyzing the BPNN used in the above approach.

\subsection{Relationship between Learning Parameters and Learning Convenience}

We use different values of learning rate $\eta$ and momentum rate $\alpha$ to train the same BPNN by using seven pairs of $P$ arrival and background noise segments (Figure 3 ). Table 1 shows the iteration number required in training this BPNN as a function of $\eta$ and $\alpha$, but with the same pattern error threshold (0.0001) and system error threshold $(0.00001)$ which are used to terminate the training procedure. A relationship between the iteration number and $\eta$ and $\alpha$ can be obtained from this table:

$$
\text { Iteration_Number }=I N_{0} * \frac{(1-\alpha)}{10 * \eta},
$$

where $I N_{0}$ is the iteration number at $\eta=0.1$ and $\alpha=0.0$. In order to verify this equation, we calculate the iteration number by using this equation with the same $I N_{0}$ (Table 1). The calculated iteration numbers fit well with the real numbers. For example, the maximum relative error is $10 \%$ at $\alpha=0.9$ and $\eta=0.9$ with an absolute error of 8 iterations.

This equation shows that: (1) the iteration number is inversely proportional to $\alpha$. Note that $\alpha$ must be less than 1.0 , otherwise the learning procedure does not converge. (2) the iteration number is the reciprocal of the $\eta$, and naturally, $\eta$ cannot be zero. Due to the nature of the reciprocal function, large $\eta$ cannot reduce the iteration number too much. For example, when $\eta$ is increased from 0.1 to 0.2 , the iteration number is reduced by $50 \%$, but when $\eta$ is increased from 0.7 to 0.8 , the iteration number is reduced by $12.5 \%$.

The iteration number is also related to the system error. It seems that the deviation of iteration number is

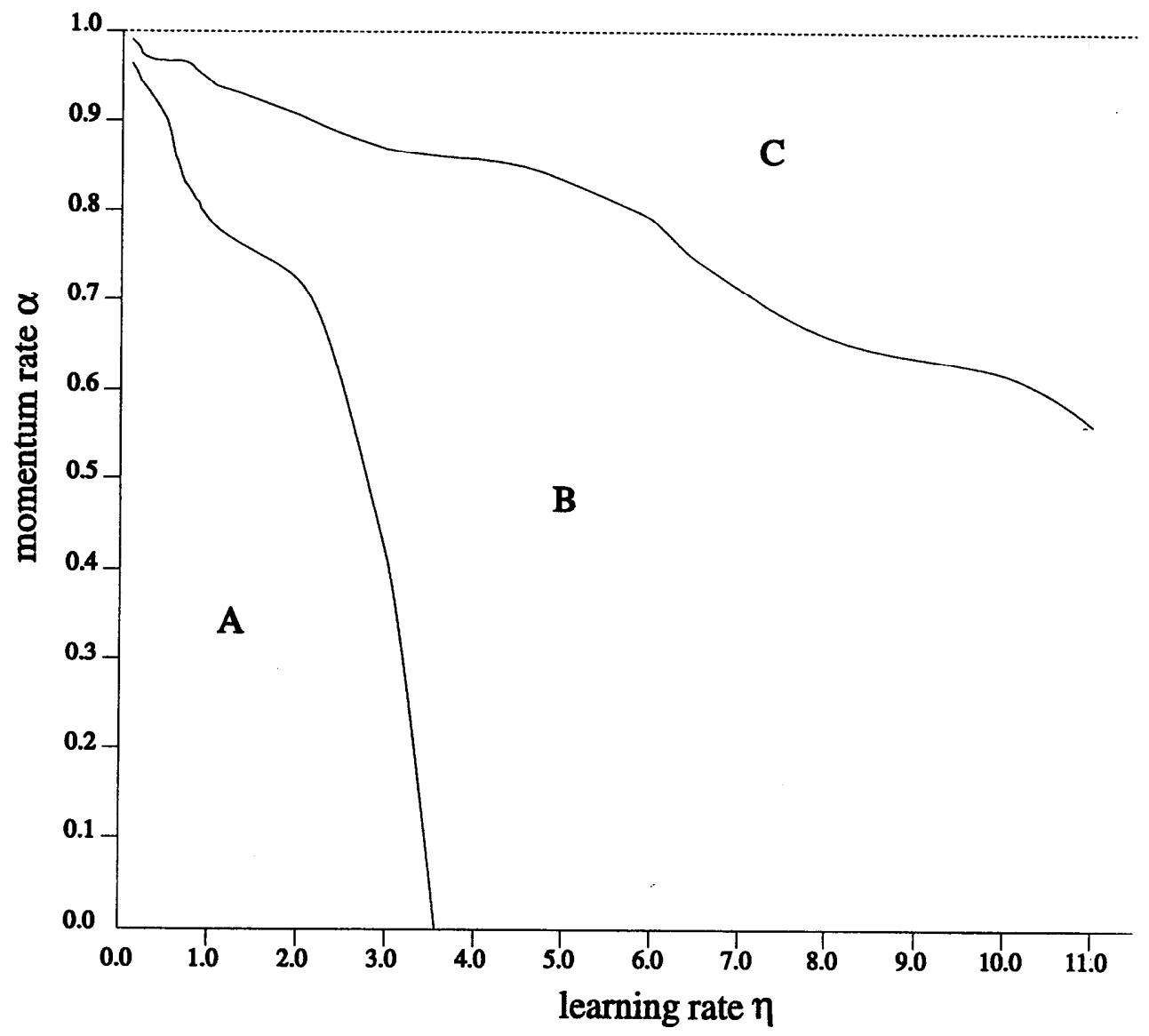

FIGURE 4. The space of $\eta$ and $\alpha$ for a BPNN with seven training datasets. A is the stable area where the fitness between real iteration number and the one calculated is accurate and the system error is stable with a relative change of less than $10 \%$; $B$ is the unstable area where the fitness is not accurate and the system error is unstable; and $C$ is the misfit area where the learning does not converge or take too many iterations, and the system error is unstable. 
TABLE 2

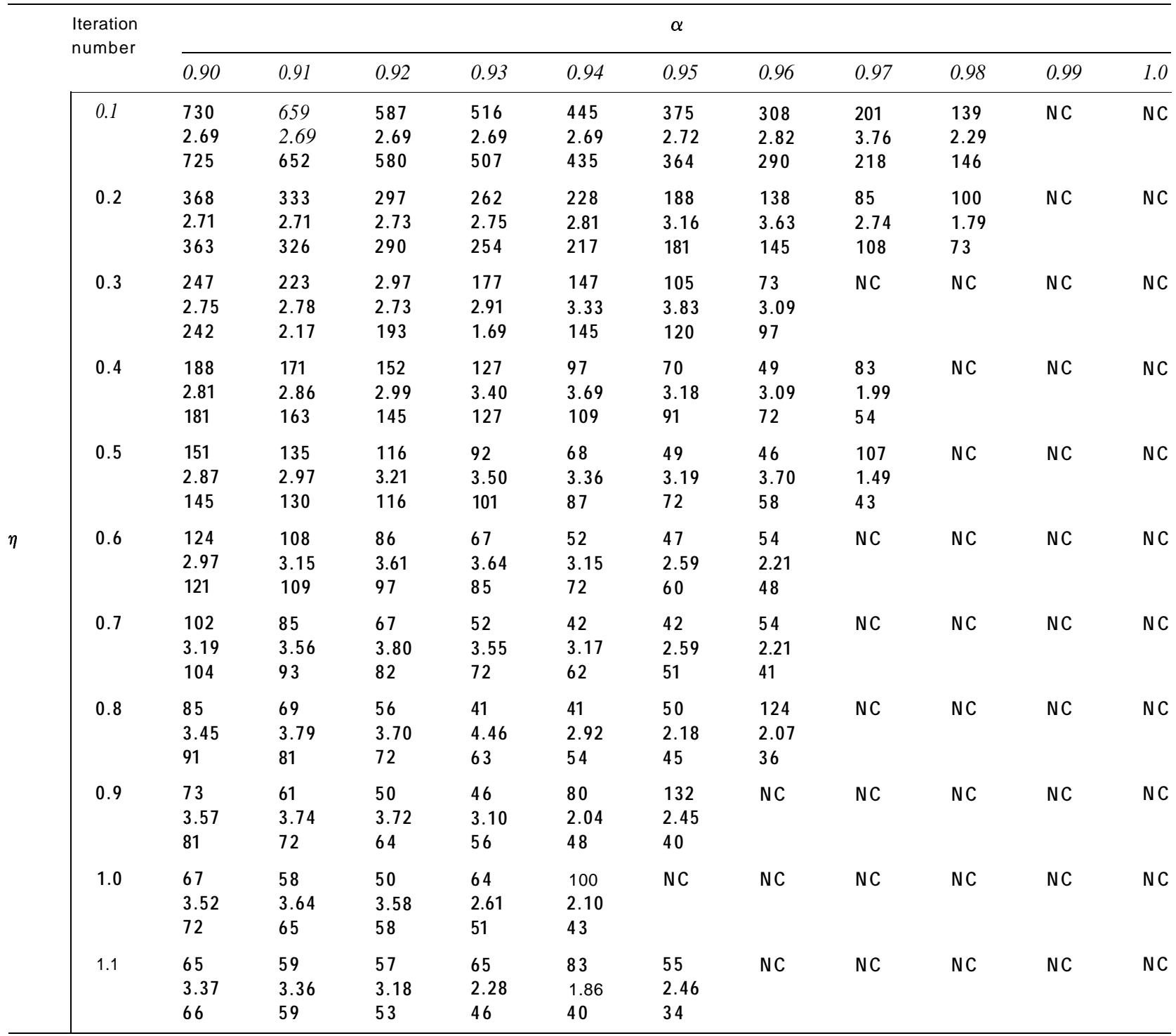

The iteration number for training with different learning rate $\eta$ ranged from 0.1 to 1.1 and momentum rate $\alpha$ ranged from 0.9 to 1.0 for the same BPNN as in Table 1. Notations are the same as in Table 1.

proportional to the deviation of the system errors related to that at $\eta=0.1$ and $\alpha=0.0$. In the training procedure, we have mainly used the pattern error to terminate the training procedure. The training procedure stops when all training patterns have an error less than 0.0001 , but the system error, varying with different learning parameters, does not need to reach its threshold (0.00001). A small system error corresponds to a long learning procedure. It seems that a better fit between the real and calculated data is obtained if the same system error is used to terminate the training procedure.

The above results only limit $\eta<1$.1. In order to obtain a complete image of the effect of $\eta$ and $\alpha$, we have examined the details of fitness between eqn (2) and the real iteration on the above BPNN when $\eta>1.1$ and $\alpha$ varies between 0.9 and 1.0 at small intervals. Tables 2 and 3 show the results in which, as the $\eta$ and $\alpha$ increase, the system error unstably deviates from the system error at $\alpha=0.0$ and $\eta=0.1$ and the real iteration number also deviates form the calculated one, and as $\eta$ increases, the value of $\alpha$, when the non-convergence occurs is decreased. The iteration number and system error, as the functions of $\alpha$ and $\eta$, can be divided into three areas (Figure 4): $\mathrm{A}$ is the stable area where the fitness between real iteration number and calculated one is accurate and the system error is stable; $B$ is the unstable area where the fitness is not accurate and the system error is unstable; and $\mathrm{C}$ is the misfit area where the learning does not converge or take too many iterations, and the system error is unstable.

\subsection{Analysis about other BPNNS}

Although the performance of this BPNN is good, we still 
TABLE 3

\begin{tabular}{|c|c|c|c|c|c|c|c|c|c|c|c|}
\hline \multirow{2}{*}{$\begin{array}{l}\text { Iteration } \\
\text { number }\end{array}$} & \multicolumn{11}{|c|}{$\alpha$} \\
\hline & 0.0 & 0.1 & 0.2 & 0.3 & 0.4 & 0.5 & 0.6 & 0.7 & 0.8 & 0.9 & 1.0 \\
\hline \multirow[t]{3}{*}{0.1} & 7248 & 6523 & 5799 & 5074 & 4350 & 3625 & 2900 & 2175 & 1451 & 730 & NC \\
\hline & 2.69 & 2.69 & 2.69 & 2.70 & 2.70 & 2.70 & 2.70 & 2.70 & 2.70 & 2.69 & \\
\hline & 7248 & 6523 & 5798 & 5074 & 4349 & 3624 & 2899 & 2174 & 1450 & 725 & \\
\hline \multirow[t]{3}{*}{1} & 756 & 681 & 606 & 532 & 457 & 382 & 307 & 231 & 156 & 67 & NC \\
\hline & 2.68 & 2.70 & 2.72 & 2.73 & 2.76 & 2.78 & 2.81 & 2.83 & 2.97 & 3.52 & \\
\hline & 725 & 652 & 580 & 507 & 435 & 362 & 290 & 217 & 145 & 73 & \\
\hline \multirow[t]{3}{*}{2} & 391 & 353 & 316 & 277 & 239 & 202 & 164 & 124 & 87 & 53 & NC \\
\hline & 2.62 & 2.64 & 2.67 & 2.71 & 2.75 & 2.79 & 2.83 & 2.85 & 2.87 & 2.40 & \\
\hline & 362 & 326 & 290 & 254 & 217 & 181 & 145 & 109 & 72 & 36 & \\
\hline \multirow[t]{3}{*}{3} & 258 & 234 & 211 & 188 & 163 & 136 & 109 & 84 & 46 & 42 & NC \\
\hline & 2.77 & 2.78 & 2.80 & 2.84 & 2.91 & 3.01 & 3.10 & 3.17, & 3.64 & 2.06 & \\
\hline & 242 & 216 & 192 & 108 & 144 & 120 & 96 & 72 & 48 & 24 & \\
\hline \multirow[t]{3}{*}{4} & 187 & 172 & 157 & 139 & 119 & 99 & 78 & 55 & 47 & $\mathrm{NC}$ & NC \\
\hline & 3.01 & 3.03 & 3.06 & 3.13 & 3.24 & 3.38 & 3.65 & 4.06 & 2.70 & & \\
\hline & 181 & 162 & 144 & 126 & 108 & 90 & 72 & 54 & 36 & & \\
\hline \multirow[t]{3}{*}{5} & 149 & 137 & 123 & 108 & 92 & 76 & 58 & 43 & 34 & NC & NC \\
\hline & 3.15 & 3.22 & 3.31 & 3.41 & 3.59 & 3.80 & 4.21 & 4.78 & 3.47 & & \\
\hline & 145 & 131 & 116 & 102 & 87 & 73 & 58 & 44 & 29 & & \\
\hline \multirow[t]{3}{*}{6} & 124 & 113 & 101 & 88 & 74 & 66 & 68 & 57 & 51 & NC & NC \\
\hline & 3.30 & 3.40 & 3.48 & 3.66 & 4.06 & 4.06 & 3.03 & 2.88 & 2.30 & & \\
\hline & 121 & 109 & 97 & 85 & 73 & 60 & 48 & 36 & 24 & & \\
\hline \multirow[t]{3}{*}{7} & 107 & 96 & 85 & 74 & 62 & 67 & 44 & 54 & NC & NC & NC \\
\hline & 3.41 & 3.50 & 3.83 & 4.34 & 4.94 & 3.62 & 5.48 & 1.68 & & & \\
\hline & 104 & 94 & 83 & 73 & 62 & 52 & 41 & 31 & & & \\
\hline \multirow[t]{3}{*}{8} & 93 & 88 & 74 & 66 & 57 & 65 & 82 & 45 & NC & $\mathrm{NC}$ & NC \\
\hline & 3.65 & 3.88 & 4.42 & 5.04 & 5.47 & 3.95 & 2.59 & 4.01 & & & \\
\hline & 91 & 82 & 73 & 64 & 55 & 46 & 36 & 27 & & & \\
\hline \multirow[t]{3}{*}{9} & 86 & 81 & 73 & 68 & 71 & 58 & 118 & 48 & NC & NC & NC \\
\hline & 4.09 & 3.81 & 4.45 & 5.03 & 4.00 & 3.26 & 1.95 & 1.73 & & & \\
\hline & 81 & 73 & 65 & 57 & 49 & 40 & 32 & 24 & & & \\
\hline \multirow[t]{3}{*}{10} & 83 & 80 & 73 & 66 & 73 & 54 & 45 & NC & NC & NC & NC \\
\hline & 4.19 & 3.79 & 3.54 & 4.01 & 3.37 & 4.56 & 3.14 & & & & \\
\hline & 73 & 65 & 60 & 52 & 45 & 37 & 30 & & & & \\
\hline \multirow[t]{3}{*}{11} & 76 & 70 & 71 & 89 & 118 & 48 & 87 & NC & NC & NC & NC \\
\hline & 4.22 & 3.80 & 3.74 & 3.94 & 2.24 & 3.42 & 1.88 & & & & \\
\hline & 66 & 57 & 51 & 44 & 38 & 31 & 24 & & & & \\
\hline
\end{tabular}

The iteration number for training with different learning rate $\eta$ ranged from and 1.0 to 11.0 with an interval of 1.0 and momentum rate $\alpha$ ranged from 0.0 to 1.0 for the same BPNN as in Table 1. Notations are the same as in Table 1.

seek the possibility to improve its performance by adding new training data. A new BPNN is finally trained by nine pairs of $P$-arrival and background noise segments (Figure 3 ) and has an improved performance than the original one (Dai and MacBeth, 1995). Here Tables 4-6 shows the iteration numbers required in training this BPNN. These tables also show the prediction from eqn (2) which provides a satisfactory fit. It also shows that the system error is more stable for this BPNN. Figure 5 shows the same features of $\eta$ and $\alpha$ as Figure 4. It seems that this better fit corresponds to the improved performance of the later BPNN.

We also examine another BPNN which is used to pick seismic arrivals from vertical component seismograms
(Dai and MacBeth, 1994b). In this case the BPNN also has three layers, but with 40 input nodes. To train this BPNN, 10 pairs of $P$-arrival and background noise segments of absolute values of vertical component recordings are selected (Figure 6). Tables 7-9 and Figure 7 show the results. Due to an unstable system error, its fit is not as good as that of the two previous BPNNs.

\subsection{Discussion}

By analyzing three BPNNs, we obtain a general relationship (eqn (2)) between the learning parameters ( $\eta$ and $\alpha$ ) and the iteration number. The iteration number depends on two factors: the system error and the learning 
TABLE 4

\begin{tabular}{|c|c|c|c|c|c|c|c|c|c|c|c|}
\hline \multirow{2}{*}{$\begin{array}{l}\text { Iteration } \\
\text { number }\end{array}$} & \multirow[b]{2}{*}{0.0} & \multirow[b]{2}{*}{0.1} & \multirow[b]{2}{*}{0.2} & \multirow[b]{2}{*}{0.3} & \multirow[b]{2}{*}{0.4} & \multirow[b]{2}{*}{0.5} & \multirow[b]{2}{*}{0.6} & \multirow[b]{2}{*}{0.7} & \multirow[b]{2}{*}{0.8} & \multirow[b]{2}{*}{0.9} & \multirow[b]{2}{*}{1.0} \\
\hline & & & & & & & & & & & \\
\hline \multirow[t]{3}{*}{0.1} & 8991 & 8096 & 7201 & 6304 & 5406 & 4507 & 3606 & 2703 & 1799 & 893 & NC \\
\hline & 1.04 & 1.04 & 1.04 & 1.04 & 1.04 & 1.04 & 1.04 & 1.04 & 1.04 & 1.03 & \\
\hline & 8991 & 8092 & 7192 & 6294 & 5396 & 4496 & 3596 & 2697 & 1798 & 899 & \\
\hline \multirow[t]{3}{*}{0.2} & 4483 & 4046 & 3596 & 3150 & 2702 & 2253 & 1803 & 1351 & 898 & 443 & NC \\
\hline & 1.05 & 1.05 & 1.04 & 1.04 & 1.04 & 1.04 & 1.04 & 1.04 & 1.03 & 1.02 & \\
\hline & 4496 & 4046 & 3596 & 3152 & 2698 & 2248 & 1798 & 1349 & 899 & 450 & \\
\hline \multirow[t]{3}{*}{0.3} & 2981 & 2688 & 2394 & 2098 & 1800 & 1502 & 1202 & 900 & 596 & 291 & NC \\
\hline & 1.05 & 1.05 & 1.05 & 1.05 & 1.05 & 1.04 & 1.04 & 1.04 & 1.03 & 1.01 & \\
\hline & 2997 & 2697 & 2397 & 2101 & 1798 & 1499 & 1199 & 899 & 599 & 300 & \\
\hline \multirow[t]{3}{*}{0.4} & 2230 & 2012 & 1792 & 1572 & 1349 & 1126 & 901 & 674 & 445 & 213 & NC \\
\hline & 1.05 & 1.05 & 1.05 & 1.05 & 1.05 & 1.04 & 1.04 & 1.04 & 1.03 & 1.01 & \\
\hline & 2248 & 2023 & 1798 & 1576 & 1349 & 1124 & 899 & 674 & 450 & 225 & \\
\hline \multirow[t]{3}{*}{0.5} & 1779 & 1606 & 1431 & 1256 & 1078 & 900 & 720 & 538 & 354 & 165 & NC \\
\hline & 1.06 & 1.05 & 1.05 & 1.05 & 1.05 & 1.05 & 1.04 & 1.04 & 1.03 & 1.03 & \\
\hline & 1798 & 1618 & 1438 & 1261 & 1079 & 899 & 719 & 538 & 360 & 180 & \\
\hline \multirow[t]{3}{*}{0.6} & 1479 & 1335 & 1191 & 1045 & 898 & 749 & 599 & 447 & 294 & 135 & NC \\
\hline & 1.06 & 1.06 & 1.05 & 1.05 & 1.05 & 1.05 & 1.05 & 1.04 & 1.04 & 1.08 & \\
\hline & 1499 & 1349 & 1199 & 1051 & 899 & 749 & 599 & 450 & 300 & 150 & \\
\hline \multirow[t]{3}{*}{0.7} & 1264 & 1142 & 1019 & 894 & 768 & 641 & 513 & 383 & 251 & 112 & NC \\
\hline & 1.06 & 1.06 & 1.06 & 1.06 & 1.06 & 1.05 & 1.05 & 1.04 & 1.03 & 1.16 & \\
\hline & 1284 & 1156 & 1027 & 899 & 770 & 642 & 513 & 385 & 257 & 128 & \\
\hline \multirow[t]{3}{*}{0.8} & 1104 & 997 & 890 & 781 & 671 & 561 & 448 & 334 & 219 & 96 & NC \\
\hline & 1.07 & 1.06 & 1.06 & 1.06 & 1.06 & 1.05 & 1.05 & 1.05 & 1.04 & 1.23 & \\
\hline & 1123 & 1011 & 899 & 788 & 674 & 562 & 450 & 337 & 225 & 112 & \\
\hline \multirow[t]{3}{*}{0.9} & 980 & 885 & 790 & 693 & 596 & 498 & 399 & 297 & 195 & 81 & NC \\
\hline & 1.07 & 1.07 & 1.06 & 1.06 & 1.06 & 1.06 & 1.05 & 1.05 & 1.04 & 1.34 & \\
\hline & 999 & 899 & 799 & 700 & 599 & 499 & 400 & 300 & 200 & 100 & \\
\hline \multirow[t]{3}{*}{1.0} & 891 & 796 & 710 & 624 & 536 & 448 & 359 & 267 & 177 & 67 & NC \\
\hline & 1.07 & 1.07 & 1.07 & 1.06 & 1.06 & 1.06 & 1.05 & 1.05 & 1.04 & 1.53 & \\
\hline & 899 & 809 & 719 & 630 & 540 & 450 & 360 & 270 & 180 & 90 & \\
\hline \multirow[t]{3}{*}{1.1} & 802 & 723 & 645 & 567 & 488 & 408 & 326 & 244 & 164 & 64 & NC \\
\hline & 1.07 & 1.07 & 1.07 & 1.07 & 1.06 & 1.06 & 1.06 & 1.05 & 1.04 & 1.58 & \\
\hline & 817 & 736 & 654 & 573 & 490 & 409 & 327 & 245 & 164 & 82 & \\
\hline
\end{tabular}

The iteration number for training with different learning rate $\eta$ ranged from 0.1 to $1 . \mathrm{l}$ and momentum rate $\alpha$ ranged from 0.0 to 1.0 . This BPNN is trained with nine pairs of P-arrival and background noise segments. Notations are the same as in Table 1.

parameters $(\eta$ and $\alpha$ ). The fit between the real number and the calculated number is related to the system error change. The system error is affected by the learning parameters, however, it seems that there is no regular rule. Although for a well trained BPNN, it should be stable. The learning parameters have only a minor effect on the fitness when $\eta$ and $\alpha$ in the stable area are small. In contrast, when the $\eta$ and $\alpha$ are large, the effect is also large. The differences increase as $\eta$ increases. For example, on the column $\alpha=0.1$ of Table 1 , although the system errors are the same when $\eta=0.1$ and $\eta=1.1$, the real iteration number at $\eta=1.1$ is still larger than the calculated one. This reflects the fact that a large deviation can take place in the weight space with a high $\eta$ in the training procedure (Pao, 1988). During the learning procedure with high $\eta$, the weight changes might rise above the desired weight state and necessitate a research. As a result, the learning procedure requires more iterations to reach this state. Although using the momentum term can reduce this jump, it cannot be totally overcome. Therefore, a large training parameter may not necessarily result in a short learning procedure.

The space of $\eta$ and $\alpha$ can be divided into three areas for the iteration number and system error (Figs 4 and 5 and Figure 7). It seems the shape of the areas reflects the BPNN's performance. For a BPNN with good performance, the stable area (A) should be large as in Figure 5. However, the other two BPNNs have a smaller stable area with lower performance. This may be used to check the performance during training of a BPNN. In addition, when using large $\eta$ and $\alpha$ to train a BPNN, the iteration number should not deviate far from the 
TABLE 5

\begin{tabular}{|c|c|c|c|c|c|c|c|c|c|c|c|}
\hline \multirow{2}{*}{$\begin{array}{l}\text { Iteration } \\
\text { number }\end{array}$} & \multicolumn{11}{|c|}{$\alpha$} \\
\hline & 0.9 & 0.91 & 0.92 & 0.93 & 0.94 & 0.95 & 0.96 & 0.97 & 0.98 & 0.99 & 1.0 \\
\hline \multirow[t]{3}{*}{0.1} & 893 & 803 & 712 & 621 & 529 & 433 & 326 & 195 & 106 & 786 & NC \\
\hline & 1.03 & 1.03 & 1.03 & 1.03 & 1.03 & 1.03 & 1.05 & 1.36 & 1.63 & 0.96 & \\
\hline & 899 & 809 & 719 & 629 & 539 & 450 & 360 & 270 & 180 & 90 & \\
\hline \multirow[t]{3}{*}{0.2} & 443 & 398 & 351 & 303 & 251 & 194 & 134 & 89 & 246 & NC & NC \\
\hline & 1.02 & 1.02 & 1.02 & 1.02 & 1.03 & 1.14 & 1.43 & 1.65 & 0.97 & & \\
\hline & 450 & 405 & 360 & 315 & 270 & 225 & 180 & 135 & 90 & & \\
\hline \multirow[t]{3}{*}{0.3} & 291 & 259 & 226 & 190 & 151 & 102 & 66 & 72 & 187 & NC & NC \\
\hline & 1.01 & 1.01 & 1.01 & 1.04 & 1.17 & 1.45 & 1.87 & 1.35 & 2.27 & & \\
\hline & 300 & 270 & 240 & 210 & 180 & 150 & 120 & 90 & 60 & & \\
\hline \multirow[t]{3}{*}{0.4} & 213 & 187 & 159 & 128 & 91 & 63 & 51 & 47 & 451 & NC & NC \\
\hline & 1.01 & 1.01 & 1.05 & 1.15 & 1.35 & 1.65 & 1.47 & 1.74 & 1.92 & & \\
\hline & 225 & 203 & 180 & 158 & 134 & 112 & 89 & 67 & 44 & & \\
\hline \multirow[t]{3}{*}{0.5} & 165 & 143 & 119 & 92 & 68 & 56 & 49 & 66 & $\mathrm{NC}$ & NC & NC \\
\hline & 1.03 & 1.07 & 1.16 & 1.30 & 1.53 & 1.57 & 1.68 & 1.71 & & & \\
\hline & 180 & 162 & 144 & 126 & 108 & 90 & 72 & 54 & & & \\
\hline \multirow[t]{3}{*}{0.6} & 135 & 116 & 94 & 74 & 59 & 50 & 34 & 477 & $\mathrm{NC}$ & $\mathrm{NC}$ & NC \\
\hline & 1.08 & 1.16 & 1.30 & 1.52 & 1.82 & 1.52 & 4.01 & 1.91 & & & \\
\hline & 150 & 135 & 120 & 105 & 90 & 75 & 60 & 45 & & & \\
\hline \multirow[t]{3}{*}{0.7} & 112 & 96 & 84 & 61 & 60 & 50 & 107 & NC & $\mathrm{NC}$ & $\mathrm{NC}$ & NC \\
\hline & 1.16 & 1.26 & 1.39 & 1.88 & 1.57 & 2.55 & 1.39 & & & & \\
\hline & 128 & 115 & 102 & 90 & 77 & 64 & 51 & & & & \\
\hline \multirow[t]{3}{*}{0.8} & 96 & 83 & 60 & 57 & 62 & 75 & 95 & 603 & $\mathrm{NC}$ & $\mathrm{NC}$ & NC \\
\hline & 1.23 & 1.37 & 1.90 & 1.84 & 1.66 & 1.25 & 1.52 & 0.98 & & & \\
\hline & 112 & 101 & 90 & 78 & 67 & 56 & 45 & 34 & & & \\
\hline \multirow[t]{3}{*}{0.9} & 81 & 63 & 53 & 57 & 63 & 39 & 36 & 467 & $\mathrm{NC}$ & $\mathrm{NC}$ & NC \\
\hline & 1.34 & 1.72 & 2.17 & 2.01 & 1.57 & 1.57 & 2.08 & 2.04 & & & \\
\hline & 100 & 90 & 80 & 70 & 60 & 50 & 40 & 30 & & & \\
\hline \multirow[t]{3}{*}{1.0} & 67 & 56 & 51 & 67 & 40 & 34 & 60 & 708 & $\mathrm{NC}$ & NC & NC \\
\hline & 1.53 & 1.99 & 2.56 & 1.40 & 1.73 & 1.80 & 1.73 & 2.20 & & & \\
\hline & 90 & 81 & 72 & 63 & 54 & 45 & 36 & 27 & & & \\
\hline \multirow[t]{3}{*}{1.1} & 64 & 57 & 52 & 69 & 37 & 36 & 96 & NC & $\mathrm{NC}$ & NC & NC \\
\hline & 1.58 & 2.04 & 2.55 & 1.37 & 1.82 & 2.10 & 1.67 & & & & \\
\hline & 82 & 74 & 66 & 57 & 49 & 41 & 37 & & & & \\
\hline
\end{tabular}

The iteration number for training with different learning rate $\eta$ ranged from 0.1 to $1 . \mathrm{l}$ and momentum rate $\alpha$ ranged from 0.9 to 1.0 for the same BPNN as in Table 4. Notations are the same as in Table 1.

predicted value. If this is so, it seems that either the BPNN is not well designed or the training dataset is not well selected.

This feature may also be used as a guide to select $\eta$ and $\alpha$ values. The $\eta$ and $\alpha$ values should be in the stable area (A) and near its boundary where the iteration number is smaller. In order to reduce the iteration number to a value as small as possible, the optimum choice for $\eta$ is 0.7 and for $\alpha$ is between 0.8 and 0.9 (must be less than 0.9 ), or 0.6 and 0.9 for a well designed BPNN.

\section{WEIGHT PATTERN ANALYSIS}

The above analysis shows that learning parameters $(\eta$ and $\alpha$ ) greatly affect the convergent speed of the learning procedure. Here we will examine their effect on the
BPNN structure. In order to examine this effect, we introduce a weight map of the BPNN structure which is produced to show the BPNN weight patterns, then analyzes this weight pattern.

\subsection{Weight Map}

The weight map of a BPNN structure shows the magnitudes of each weight connecting to the different layers in the BPNN. Figure 8 shows an example of a weight map of a BPNN trained with nine pairs of training dataset. For this three-layer BPNN, this map includes two plots: (1) the mapping of weights between the input layer and the hidden layer; and (2) the mapping between the hidden layer and the output layer. In each mapping, solid circles are shaded on a grey scale corresponding to a magnitude 
TABLE 6

\begin{tabular}{|c|c|c|c|c|c|c|c|c|c|c|c|}
\hline \multirow{2}{*}{$\begin{array}{l}\text { Iteration } \\
\text { number }\end{array}$} & \multicolumn{11}{|c|}{$\alpha$} \\
\hline & 0.0 & 0.1 & 0.2 & 0.3 & 0.4 & 0.5 & 0.6 & 0.7 & 0.8 & 0.9 & 1.0 \\
\hline \multirow[t]{3}{*}{0.1} & 8991 & 8096 & 7201 & 6304 & 5406 & 4507 & 3606 & 2703 & 1799 & 893 & NC \\
\hline & 1.04 & 1.04 & 1.04 & 1.04 & 1.04 & 1.04 & 1.04 & 1.04 & 1.04 & 1.03 & \\
\hline & 8991 & 8092 & 7192 & 6294 & 5396 & 4496 & 3596 & 2697 & 1798 & 899 & \\
\hline \multirow[t]{3}{*}{1.0} & 891 & 796 & 710 & 624 & 536 & 448 & 359 & 267 & 177 & 67 & NC \\
\hline & 1.07 & 1.07 & 1.07 & 1.06 & 1.06 & 1.06 & 1.05 & 1.05 & 1.04 & 1.53 & \\
\hline & 899 & 809 & 719 & 630 & 540 & 450 & 360 & 270 & 180 & 90 & \\
\hline \multirow[t]{3}{*}{2.0} & 475 & 423 & 374 & 326 & 281 & 230 & 196 & 149 & 97 & 122 & NC \\
\hline & 1.05 & 1.06 & 1.06 & 1.06 & 1.05 & 1.05 & 1.03 & 1.02 & 1.11 & 1.04 & \\
\hline & 450 & 405 & 360 & 315 & 270 & 225 & 180 & 135 & 90 & 45 & \\
\hline \multirow[t]{3}{*}{3.0} & 342 & 305 & 271 & 240 & 211 & 181 & 151 & 133 & 101 & 34 & NC \\
\hline & 1.06 & 1.06 & 1.06 & 1.06 & 1.06 & 1.06 & 1.09 & 1.15 & 1.50 & 2.16 & \\
\hline & 300 & 270 & 240 & 210 & 180 & 150 & 120 & 90 & 60 & 30 & \\
\hline \multirow[t]{3}{*}{4.0} & 263 & 239 & 218 & 198 & 179 & 161 & 147 & 91 & 33 & 245 & NC \\
\hline & 1.16 & 1.16 & 1.16 & 1.16 & 1.16 & 1.19 & 1.22 & 1.34 & 2.43 & 0.91 & \\
\hline & 2.25 & 2.03 & 180 & 158 & 135 & 113 & 90 & 68 & 45 & 23 & \\
\hline \multirow[t]{3}{*}{5.0} & 213 & 196 & 183 & 175 & 164 & 135 & 112 & 73 & 100 & NC & NC \\
\hline & 1.26 & 1.27 & 1.27 & 1.25 & 1.24 & 1.33 & 1.42 & 1.61 & 1.73 & & \\
\hline & 180 & 162 & 144 & 126 & 108 & 90 & 72 & 54 & 36 & & \\
\hline \multirow[t]{3}{*}{6.0} & 184 & 171 & 167 & 167 & 146 & 120 & 126 & 58 & 68 & NC & NC \\
\hline & 1.33 & 1.35 & 1.31 & 1.26 & 1.39 & 1.64 & 1.65 & 1.81 & 2.64 & & \\
\hline & 150 & 135 & 120 & 105 & 90 & 75 & 60 & 45 & 30 & & \\
\hline \multirow[t]{3}{*}{7.0} & 164 & 156 & 164 & 156 & 129 & 104 & 80 & 108 & 57 & NC & NC \\
\hline & 1.38 & 1.42 & 1.39 & 1.51 & 1.74 & 2.00 & 1.83 & 1.81 & 1.66 & & \\
\hline & 128 & 115 & 102 & 90 & 77 & 64 & 51 & 38 & 26 & & \\
\hline \multirow[t]{3}{*}{8.0} & 147 & 144 & 155 & 135 & 125 & 40 & 57 & 57 & NC & NC & NC \\
\hline & 1.52 & 1.65 & 1.60 & 2.01 & 1.42 & 2.37 & 2.91 & 2.91 & & & \\
\hline & 112 & 101 & 90 & 67 & 56 & 45 & 34 & 34 & & & \\
\hline \multirow[t]{3}{*}{9.0} & 125 & 103 & 128 & 123 & 101 & 81 & 81 & 45 & NC & NC & NC \\
\hline & 1.50 & 1.93 & 1.77 & 1.50 & 2.48 & 1.51 & 1.63 & 2.45 & & & \\
\hline & 100 & 90 & 80 & 70 & 60 & 50 & 40 & 30 & & & \\
\hline \multirow[t]{3}{*}{10.0} & 113 & 127 & 116 & 114 & 110 & 71 & 45 & 327 & NC & NC & NC \\
\hline & 1.46 & 1.31 & 2.20 & 1.52 & 2.05 & 2.55 & 1.67 & 1.40 & & & \\
\hline & 90 & 81 & 72 & 63 & 54 & 45 & 36 & & & & \\
\hline \multirow[t]{3}{*}{11.0} & 126 & 105 & 93 & 72 & 86 & 40 & 72 & 312 & NC & NC & NC \\
\hline & 1.94 & 2.58 & 1.43 & 2.50 & 1.67 & 3.44 & 2.23 & 1.52 & & & \\
\hline & 82 & 74 & 66 & 57 & 49 & 41 & 33 & 25 & & & \\
\hline
\end{tabular}

The iteration number for training with different learning rate $\eta$ ranged from 1.0 to 11.0 with an interval of 1.0 and momentum rate $\alpha$ ranged from 0.0 to 1.0 for the same BPNN as in Table 4. Notations are the same as in Table 1.

range indicated by the key, with the top row of circles in map (1) and far right row of circles in map (2) representing the nodal thresholds. This method can be applied to any BPNN application, enabling illumination of the "black-box" approach of BPNN analysis and examination of the BPNN function. For example, this weight map shows that the weight pattern between the input nodes and hidden nodes can be divided into two portions with different patterns at the tenth input nodes. We can expect that for the BPNNs with the same structures, if they have the same weight patterns, they should have the same performance.

Visual checking of the weight maps of BPNNs training with different parameters shows that with the same training dataset, they have similar weight patterns. The weight map between the input nodes and hidden nodes can be divided into two portions at the tenth input node. Weights connected to nodes between the first to tenth nodes show a "high contrast" pattern, in which most of the weights have large absolute values. Weights connected to nodes between the eleventh and thirtieth nodes show a "low contrast" pattern in which most of the weights have small absolute values. Although a few weights show some changes as $\eta$ and $\alpha$ alter, the whole pattern is similar. Therefore, these trained BPNNs with similar weight patterns should have similar performances. 


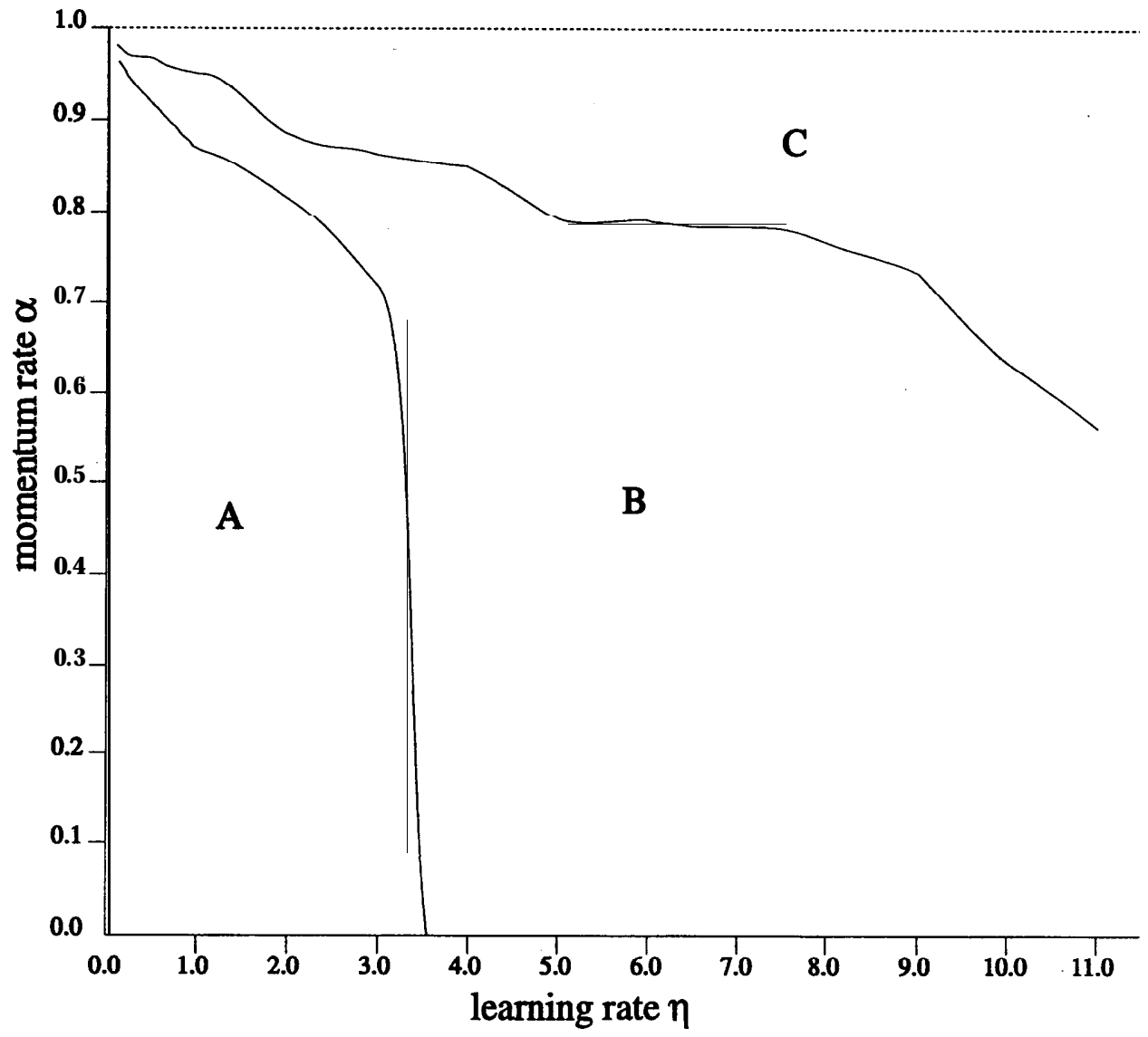

FIGURE 5. The space of $\eta$ and $\alpha$ for a BPNN with nine training datasets. Notations as in Figure 4.

\subsection{Weight Pattern Analysis}

In order to understand how the trained BPNN picks seismic arrivals, further study is required to view the mechanism by which the trained BPNN performs its classification. Before training, the weights and thresholds in Figure 8 are randomly initialized between -0.5 and +0.5 , and after training their values range from -2.12 to +2.02 . It appears that the pattern of weights connecting the input layer to the hidden layer is divided into two portions at the tenth sample, corresponding to the $P$-arrival onset in the input signal in which before the onset the signal energy is low and after the onset the signal energy is high. The first portion between the first and tenth nodes shows a "high contrast" weight pattern in which the absolute values of most weights are large: most weights connected to the first and seventh hidden nodes are negative large values, and most weights connected to the fourth hidden node are positive large values. The second portion between the eleventh and thirtieth nodes shows a "low contrast" weight pattern in which the absolute values of most weights are small. The weight pattern between the hidden nodes and the output nodes is related to the pattern between the input node and hidden node. For example, the weights connected to the first and seventh hidden nodes have large negative values when they connect to the noise output node and large positive values when they connect to the $P$ output node, and the weights connected to the fourth hidden node have a large positive value when they connect to the noise node and large negative values when they connect to the $P$ node. It seems the first, fourth and seventh hidden nodes are more active in the performance because the weights assigned to them have larger values than others.

According to the sigmoidal function of nodes and the input signal which contains all positive values which are normalized between 1 and 0 , a negative weight decreases the node output and a positive weight increases the node output. In the case of a background noise segment being fed into the BPNN, because all values are high and the weights are large in the first portion and play the principal role, the weights in the second portion will be neglected, The first and seventh hidden nodes give low output and the fourth hidden node gives high output. So the final output will be (high, low) values for the noise. However, in the case of a $P$-arrival segment being fed into the BPNN, because of the low values before the $P$-onset, the weights in the first portion can be neglected. The weights in the second portion play the principal role in the BPNN output which will be (low, high) values according to the training. As the sliding window moves along the seismogram, once the onset of an arrival passes into the input window, the values of the input samples 

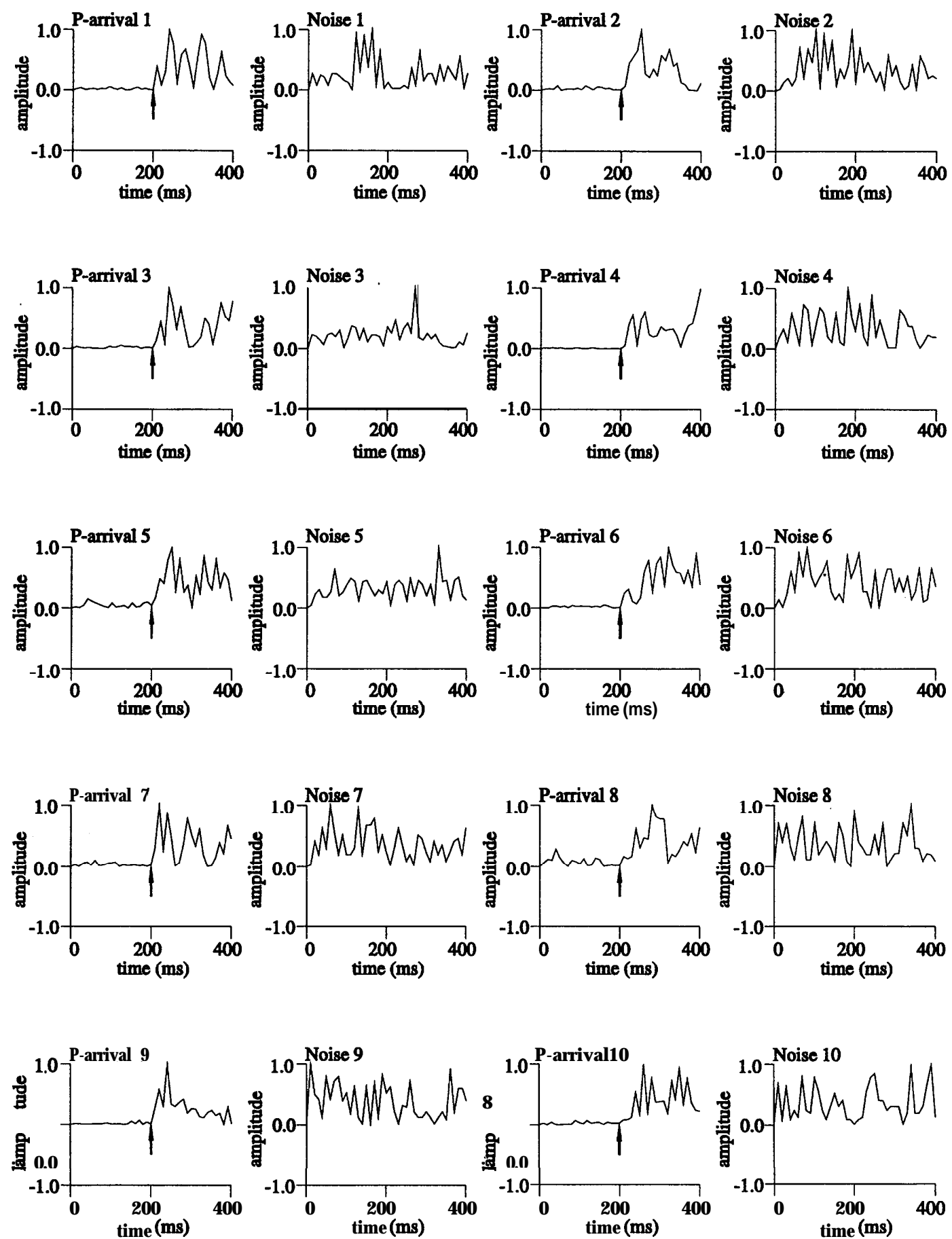

FIGURE 6. Ten pairs of P-arrival and background noise segments with absolute values of vertical component recordings used for training a BPNN. Noise segments are extracted prior to the P-arrivals in the same seismograms. Arrows on P-arrival segments indicate arrival onset times used to train the BPNN, all at the twenty-first sample. These segments are individually normalized before being input into the BPNN.

before the onset decrease, and the first portion will lose its effect on the output which attempts to reach the (low, high) state indicating a $P$-arrival. At the stage of the onset at the eleventh node, the output should reach the peak of the (low, high) state. However, as the onset moves into the position before the tenth nodes, the input values before the 10 nodes increases, so that the first portion will strengthen its effect on the output which attempts to reach the (high, low) state indicating background noise. It is too complex to show the numerical results, however, this simple analysis gives a clear image of the details of BPNN performance under different conditions.

It is interesting to note that hidden nodes have different activities according to the values of weights connected to them. It seems that active hidden nodes (e.g. the first, fourth, and seventh nodes) can recognize the main 
TABLE 7

\begin{tabular}{|c|c|c|c|c|c|c|c|c|c|c|c|}
\hline \multirow{2}{*}{$\begin{array}{l}\text { Iteration } \\
\text { number }\end{array}$} & \multirow[b]{2}{*}{0.0} & \multirow[b]{2}{*}{0.1} & \multirow[b]{2}{*}{0.2} & \multirow[b]{2}{*}{0.3} & \multirow[b]{2}{*}{0.4} & \multirow[b]{2}{*}{0.5} & \multirow[b]{2}{*}{0.6} & \multirow[b]{2}{*}{0.7} & \multirow[b]{2}{*}{0.8} & \multirow[b]{2}{*}{0.9} & \multirow[b]{2}{*}{1.0} \\
\hline & & & & & & & & & & & \\
\hline \multirow[t]{3}{*}{0.1} & 10134 & 9124 & 8116 & 7108 & 6098 & 5089 & 4080 & 3069 & 2058 & 1045 & NC \\
\hline & 2.53 & 2.53 & 2.52 & 2.52 & 2.51 & 2.50 & 2.49 & 2.47 & 2.43 & 2.31 & \\
\hline & 10134 & 9120 & 8107 & 7093 & 6080 & 5067 & 4053 & 3040 & 2026 & 1013 & \\
\hline \multirow[t]{3}{*}{0.2} & 5093 & 4588 & 4084 & 3579 & 3074 & 2569 & 2063 & 1557 & 1050 & 541 & NC \\
\hline & 2.51 & 2.50 & 2.50 & 2.49 & 2.48 & 2.46 & 2.44 & 2.40 & 2.33 & 2.12 & \\
\hline & 5067 & 4560 & 4053 & 3547 & 3040 & 2533 & 2026 & 1520 & 1013 & 506 & \\
\hline \multirow[t]{3}{*}{0.3} & 3416 & 3079 & 2741 & 2404 & 2067 & 1730 & 1393 & 1054 & 714 & 371 & NC \\
\hline & 2.49 & 2.48 & 2.47 & 2.46 & 2.44 & 2.42 & 2.38 & 2.34 & 2.25 & 1.99 & \\
\hline & 3378 & 3040 & 2702 & 2364 & 2026 & 1689 & 1351 & 1013 & 675 & 338 & \\
\hline \multirow[t]{3}{*}{0.4} & 2580 & 2326 & 2072 & 1819 & 1565 & 1312 & 1058 & 804 & 548 & 292 & NC \\
\hline & 2.47 & 2.45 & 2.44 & 2.42 & 2.40 & 2.37 & 2.33 & 2.27 & 2.15 & 1.85 & \\
\hline & 2534 & 2280 & 2026 & 1773 & 1520 & 1267 & 1013 & 760 & 506 & 253 & \\
\hline \multirow[t]{3}{*}{0.5} & 2081 & 1876 & 1672 & 1469 & 1265 & 1062 & 859 & 655 & 450 & 240 & NC \\
\hline & 2.44 & 2.43 & 2.41 & 2.39 & 2.36 & 2.32 & 2.27 & 2.19 & 2.04 & 1.76 & \\
\hline & 2027 & 1824 & 1621 & 1418 & 1216 & 1013 & 810 & 608 & 405 & 202 & \\
\hline \multirow[t]{3}{*}{0.6} & 1751 & 1579 & 1407 & 1237 & 1067 & 897 & 726 & 556 & 385 & 203 & NC \\
\hline & 2.41 & 2.39 & 2.37 & 2.35 & 2.31 & 2.27 & 2.21 & 2.12 & 1.96 & 1.71 & \\
\hline & 1689 & 1520 & 1351 & 1274 & 1013 & 844 & 675 & 506 & 338 & 169 & \\
\hline \multirow[t]{3}{*}{0.7} & 1518 & 1368 & 1219 & 1072 & 925 & 779 & 632 & 486 & 338 & 173 & NC \\
\hline & 2.37 & 2.35 & 2.34 & 2.31 & 2.27 & 2.22 & 2.15 & 2.04 & 1.88 & 1.74 & \\
\hline & 1448 & 1302 & 1158 & 1013 & 868 & 723 & 579 & 434 & 289 & 145 & \\
\hline \multirow[t]{3}{*}{0.8} & 1346 & 1212 & 1080 & 950 & 820 & 691 & 563 & 434 & 300 & 153 & NC \\
\hline & 2.32 & 2.31 & 2.29 & 2.26 & 2.22 & 2.17 & 2.08 & 1.97 & 1.84 & 1.67 & \\
\hline & 1267 & 1140 & 1013 & 886 & 760 & 633 & 506 & 380 & 253 & 126 & \\
\hline \multirow[t]{3}{*}{0.9} & 1214 & 1092 & 973 & 856 & 739 & 624 & 509 & 393 & 272 & 144 & NC \\
\hline & 2.28 & 2.27 & 2.24 & 2.21 & 2.17 & 2.10 & 2.02 & 1.91 & 1.78 & 1.53 & \\
\hline & 1126 & 1013 & 901 & 788 & 676 & 563 & 450 & 337 & 225 & 112 & \\
\hline \multirow[t]{3}{*}{1.0} & 1111 & 998 & 889 & 782 & 675 & 570 & 466 & 359 & 253 & 136 & NC \\
\hline & 2.22 & 2.21 & 2.19 & 2.16 & 2.12 & 2.05 & 1.96 & 1.86 & 1.72 & 1.52 & \\
\hline & 1013 & 912 & 811 & 711 & 608 & 507 & 409 & 304 & 203 & 101 & \\
\hline \multirow[t]{3}{*}{1.1} & 1029 & 923 & 821 & 722 & 624 & 527 & 430 & 331 & 243 & 126 & NC \\
\hline & 2.16 & 2.16 & 2.14 & 2.10 & 2.06 & 1.99 & 1.91 & 1.82 & 1.60 & 1.44 & \\
\hline & 921 & 829 & 737 & 644 & 552 & 460 & 360 & 331 & 184 & 92 & \\
\hline
\end{tabular}

The iteration number for training with different learning rate $\eta$ ranged from 0.1 to 1 .l and momentum rate $\alpha$ ranged from 0.0 to 1.0 . This BPNN is trained with 10 pairs of P-arrival and background noise segments of vertical component. Notations are the same as in Table 1.

features of an input segment and other hidden nodes may improve the BPNN performance by recognizing the subtle features of the input signal.

\subsection{Weight Pattern Analysis for Another BPNN}

For the BPNN used to pick seismic arrivals from vertical component recordings, a similar weight pattern (Figure 9) is obtained, although this BPNN with 40 input nodes was trained by 10 pairs of $P$-arrival and background noise segments.

Before training, the weights and thresholds were randomly initialized between -0.5 and +0.5 . After training, the values of weights and thresholds range from -2.41 to 2.26 . This pattern also shows that the weights connecting the input layer to the hidden layer are divided into two portions at the twentieth sample, corresponding to the $P$-arrival onset in the input signal where before the onset the signal energy is low and after the onset the signal energy is high. The first portion between the first and twentieth nodes also shows a "high contrast" weight pattern, but the second portion between the twenty-first and fortieth nodes shows a "low contrast" weight pattern. Comparing with the weight map in Figure 8, two BPNNs have similar weight patterns, although their structure and the training dataset are quite different. Their similar weight pattern means that they operate in the same way and can do the same work. The significant feature in the two weight maps is that each of them is divided into two portions at the onset of the training $P$-arrival segments. These two portions have different functions in doing the "picking work". For example, 
TABLE 8

\begin{tabular}{|c|c|c|c|c|c|c|c|c|c|c|c|}
\hline \multirow{2}{*}{$\begin{array}{l}\text { Iteration } \\
\text { number }\end{array}$} & \multicolumn{11}{|c|}{$\mathrm{a} !$} \\
\hline & 0.90 & 0.91 & 0.92 & 0.93 & 0.94 & 0.95 & 0.96 & 0.97 & 0.98 & 0.99 & 1.0 \\
\hline \multirow[t]{3}{*}{0.1} & 1045 & 944 & 843 & 742 & 641 & 541 & 443 & 368 & 215 & NC & NC \\
\hline & 2.31 & 2.28 & 2.24 & 2.19 & 2.12 & 2.02 & 1.88 & 1.63 & 2.07 & & \\
\hline & 1013 & 918 & 810 & 709 & 608 & 507 & 405 & 304 & 203 & & \\
\hline \multirow[t]{3}{*}{0.2} & 541 & 490 & 439 & 389 & 338 & 293 & 261 & 184 & 304 & NC & NC \\
\hline & 2.12 & 2.08 & 1.94 & 1.94 & 1.86 & 1.76 & 1.72 & 2.51 & 1.27 & & \\
\hline & 506 & 455 & 354 & 354 & 304 & 253 & 202 & 125 & 101 & & \\
\hline \multirow[t]{3}{*}{0.3} & 371 & 337 & 304 & 275 & 254 & 211 & 159 & 103 & 169 & NC & NC \\
\hline & 1.99 & 1.94 & 1.89 & 1.81 & 1.70 & 1.82 & 1.97 & 2.70 & 3.54 & & \\
\hline & 338 & 304 & 270 & 237 & 203 & 169 & 135 & 101 & 68 & & \\
\hline \multirow[t]{3}{*}{0.4} & 292 & 268 & 246 & 221 & 184 & 144 & 99 & 77 & 337 & NC & NC \\
\hline & 1.85 & 1.80 & 1.73 & 1.69 & 1.79 & 2.06 & 3.40 & 3.18 & 5.09 & & \\
\hline & 253 & 228 & 202 & 177 & 152 & 127 & 101 & 76 & 51 & & \\
\hline \multirow[t]{3}{*}{0.5} & 240 & 219 & 195 & 166 & 135 & 105 & 83 & 63 & NC & NC & NC \\
\hline & 1.76 & 1.72 & 1.72 & 1.78 & 1.91 & 2.53 & 2.80 & 1.77 & & & \\
\hline & 202 & 182 & 162 & 141 & 121 & 101 & 81 & 61 & & & \\
\hline \multirow[t]{3}{*}{0.6} & 203 & 181 & 154 & 128 & 104 & 83 & 63 & 86 & 506 & NC & NC \\
\hline & 1.71 & 1.72 & 1.78 & 1.75 & 1.97 & 2.33 & 2.34 & 1.71 & 1.04 & & \\
\hline & 169 & 152 & 135 & 118 & 101 & 85 & 68 & 51 & 34 & & \\
\hline \multirow[t]{3}{*}{0.7} & 173 & 150 & 129 & 107 & 90 & 70 & 89 & 424 & NC & NC & NC \\
\hline & 1.74 & 1.71 & 1.55 & 1.56 & 1.86 & 2.17 & 1.59 & 2.11 & & & \\
\hline & 145 & 131 & 126 & 102 & 87 & 73 & 58 & 44 & & & \\
\hline \multirow[t]{3}{*}{0.8} & 153 & 135 & 116 & 99 & 77 & 79 & 142 & NC & NC & NC & NC \\
\hline & 1.67 & 1.49 & 1.33 & 1.70 & 2.24 & 1.58 & 1.50 & & & & \\
\hline & 126 & 113 & 101 & 98 & 86 & 73 & 60 & & & & \\
\hline \multirow[t]{3}{*}{0.9} & 144 & 127 & 108 & 93 & 74 & 173 & NC & NC & NC & NC & NC \\
\hline & 1.53 & 1.41 & 1083 & 2.07 & 2.41 & 1.67 & & & & & \\
\hline & 112 & 101 & 90 & 78 & 67 & 56 & & & & & \\
\hline \multirow[t]{3}{*}{1.0} & 136 & 119 & 103 & 90 & 68 & 52 & 328 & NC & NC & NC & NC \\
\hline & 1.52 & 1.47 & 1.67 & 1.78 & 2.03 & 2.57 & 1.31 & & & & \\
\hline & 101 & 91 & 81 & 71 & 61 & 50 & 40 & & & & \\
\hline \multirow[t]{3}{*}{1.1} & 126 & 111 & 95 & 75 & 53 & 78 & 490 & NC & NC & NC & NC \\
\hline & 1.44 & 1.31 & 1.20 & 1.19 & 1.43 & 1.26 & 1.68 & & & & \\
\hline & 92 & 83 & 74 & 64 & 55 & 46 & 37 & & & & \\
\hline
\end{tabular}

The iteration number for training with different learning rate $\eta$ ranged from 0.1 to 1.1 and momentum rate $\alpha$ ranged from 0.9 to 1.0 for the same BPNN as in Table 7. Notations are the same as in Table 1.

the "high contrast" portion has a strong effect on picking $P$-arrival and the "low contrast" portion has a strong effect on picking background noise. The hidden nodes in this BPNN also have different activities. The first, fifth, and sixth hidden nodes are active in the performance because the weights connected to them have larger values than others. Comparing two BPNNs, the sequence number of the active hidden nodes are different. The active hidden nodes and their attributes depend on the initial values of weights which are randomly generated.

\section{SUMMARY}

In this paper, we have examined the effect of training parameter $(\eta$ and $\alpha)$ on the BPNN learning procedure and its performance. The results show that the training para- meters mainly affect the convergence of learning procedure and do not affect the structure of the trained BPNNs too greatly, therefore, these BPNNs should have similar performance. As a result, we do not need to put too much effort into selecting their optimum values in each case. This will allow us to devote our attention only to the performance of the trained BPNN and can make a complex problem simple. It is important for the case where a large BPNN and a large training dataset are involved. In order to obtain a quick learning procedure, we need to select the learning parameter to be as large as possible. However the learning procedure might be unstable and the weights of BPNN might be trapped in a local minimum by using too large a value of $\eta$ (Rumelhart et al., 1986). The suggested value for $\eta$ is 0.7 , and $\alpha$ between 0.8 and 0.9 (less than 0.9 ) or $\eta=0.6$ and $\alpha=0.9$. This is 
TABLE 9

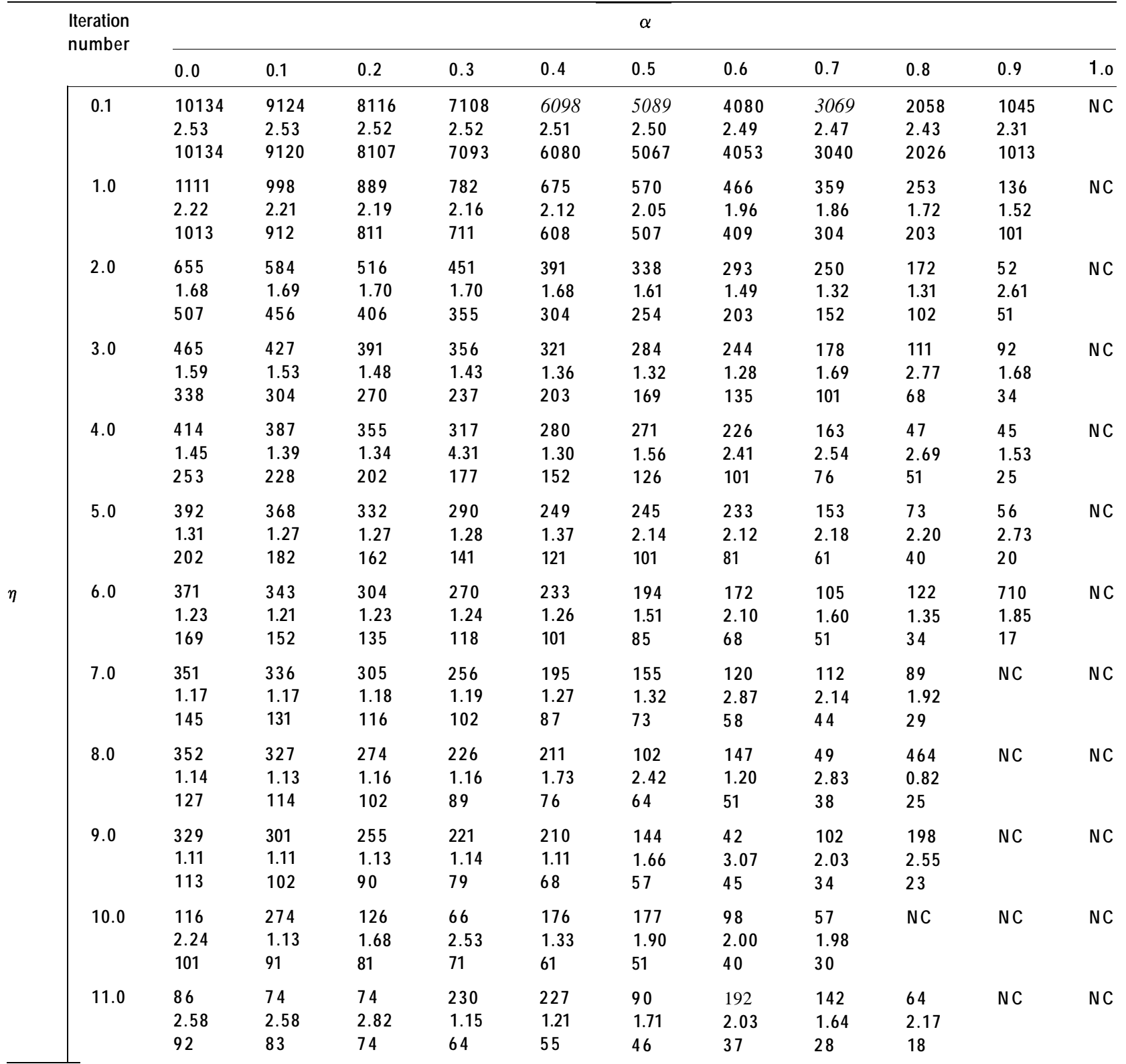

The iteration number for training with different learning rate $\eta$ ranged from 1.0 to 11.0 with an interval of 1.0 and momentum rate $\alpha$ ranged from 0.0 to 1.0 for the same BPNN as in Table 7. Notations are the same as in Table 1.

slightly different from other authors' suggestions that the suggested value is $\eta=0.7$ and $\alpha=0.9$ (McClelland and Rumelhart, 1988; Pao, 1988; Demuth and Beale, 1993).

In this paper, we use the "weight map" as a tool to investigate the interior and performance of BPNNs. For the BPNNs which are used to pick arrivals, although their structure and training dataset are different, they have similar weight patterns, which are divided into two portions corresponding to the onset of training $P$-arrivals and having different functions in the picking work. Changing learning parameters does not change the weight pattern of a trained BPNN. Although it is not possible to fully understand the logic underlying the BPNN solution by visually inspecting these weight patterns, and the weight combinations in this map are not as easily distinguished as the parameter estimates, the weight map may still lend some perspective as to which aspects of the seismic waveform which are more relevant to its solution than others, and thus be of benefit to further processing schemes or studies.

\section{REFERENCES}

Dai, H. C., \& MacBeth, C. (1994). Split shear-wave analysis using an artificial neural network. First Break, 12(12), 605-613. 


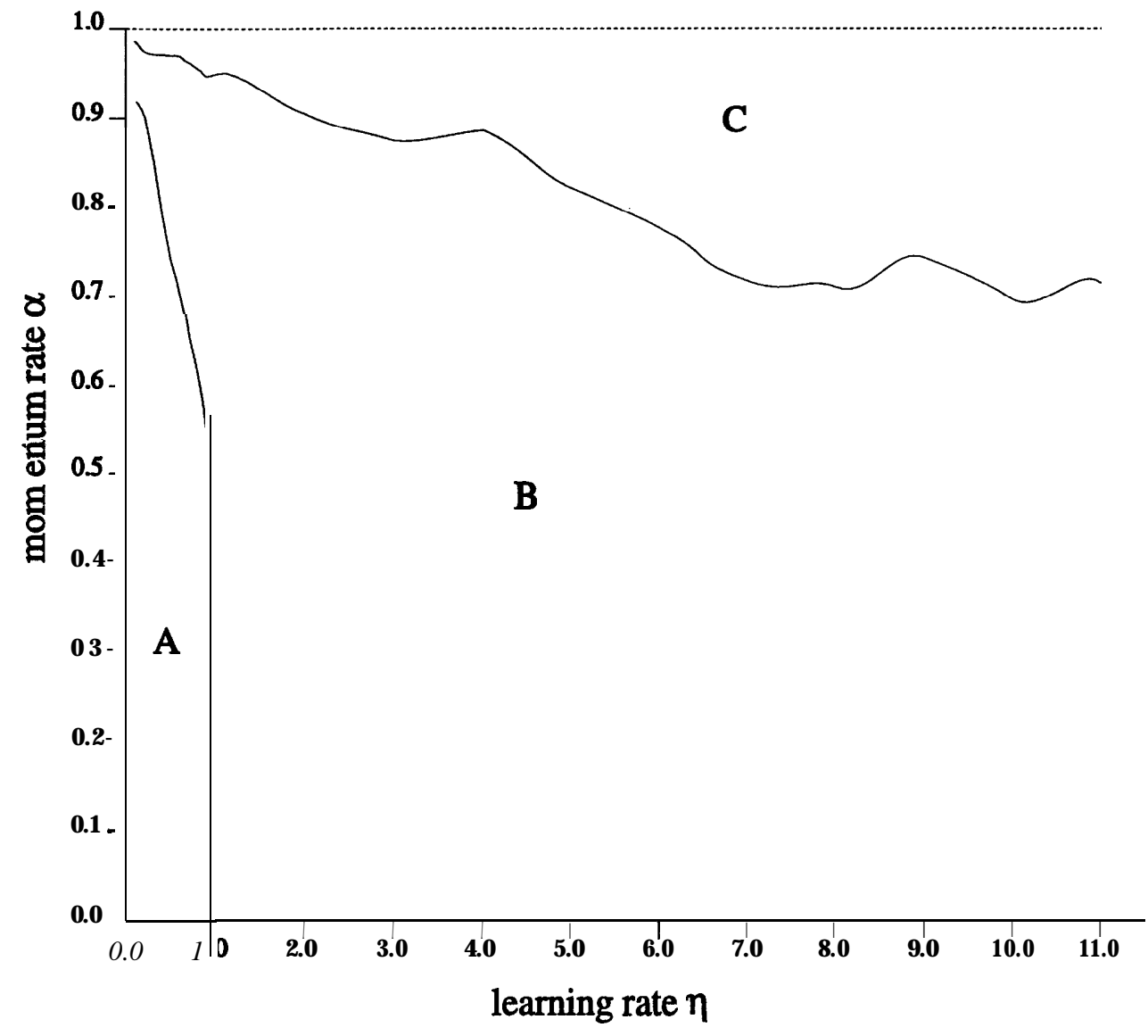

FIGURE 7. The space of $\eta$ and $\alpha$ for a BPNN with 10 training datasets. Notations as in Figure 4.

Dai, H. C. \& MacBeth, C. (1994b). Automatic picking seismic arrivals of single component recordings from local earthquake data using artificial neural networks. British Geological Survey internal report: $W L / 94 / 27$.

Dai, H. C., \& MacBeth, C. (1995). Automatic picking of seismic arrivals in local earthquake data using an artificial neural network. Geophysical Journal International, 120, 758-774.

Demuth, H. \& Beale, M. (1993). Neural network toolbox for use with MATLAB: User's guide. Natick, MA: The Math Works, Inc.

Dowla, F., Taylor, S. R., \& Anderson, R. W. (1990). Seismic discrimination with artificial neural networks: preliminary results with regional spectral data. Bulletin of the Seismological Society of America, 80, 1346- 1373 .

Fausett, L. (1994). Fundamentals of neural networks. Englewood
Cliffs, NJ: Prentice Hall.

Gorman, R. P., \& Sejnowski, T. J. (1988). Analysis of hidden units in a layered network trained to classify sonar targets. Neural Networks, $1,75-89$.

McClelland, J. \& Rumelhart, D. (1988). Explorations in parallel distributed processing, a handbook of model, program, and exercises. Cambridge, MA:The MIT Press.

McCormack, M. D. (1991). Neural computing in geophysics. Geophysics: The Leading Edge of Exploration, 10, 11-15.

Pao, Y.H. (1988). Adaptive pattern recognition and neural networks. New YorkAddison-Wesley Publishing Company, Inc.

Rumelhart, D., Hinton, G. E., \& Williams, R. J. (1986). Learning representations by back-propagating errors. Nature, 323, 533536. 
(1)

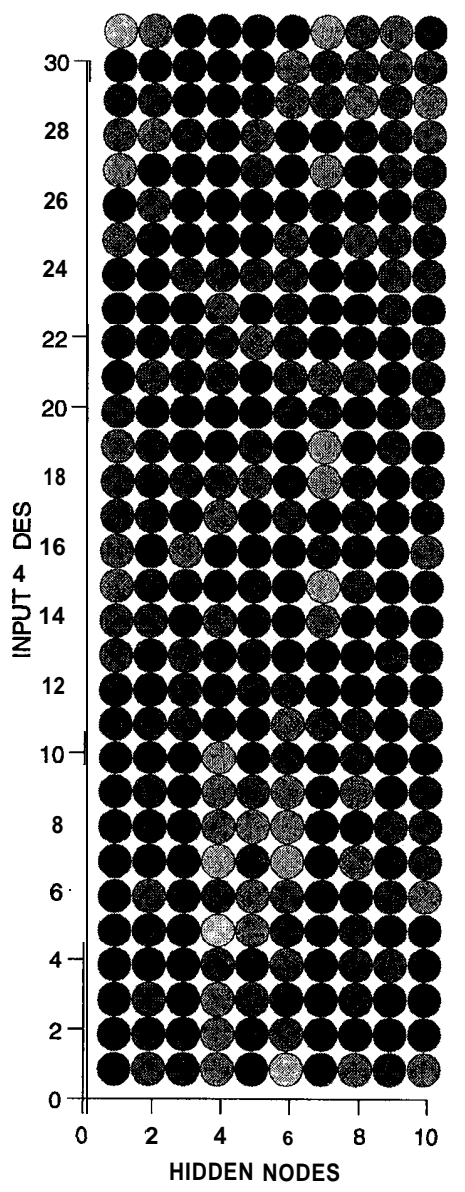

(2)

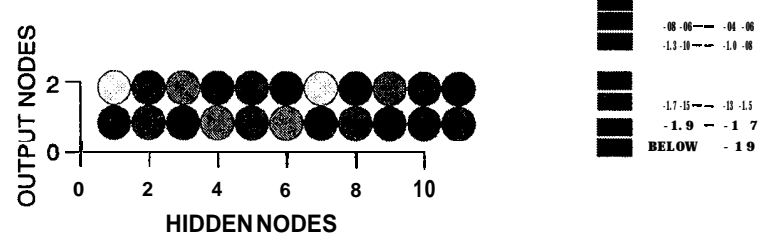

FIGURE 8. Weight map for a trained BPNN: (1) the mapping of weights between the input layer and the hidden layer; (2) the mapping between the hidden layer and the output layer. Shaded circles represent the weights and the contrast in each circle indicates the value of a weight. The top row of circles in (1) and the right row of circles in (2) represent the node thresholds. This BPNN is trained with nine pairs of $/ \mathrm{J}$-arrival and background noise segments.
(1)

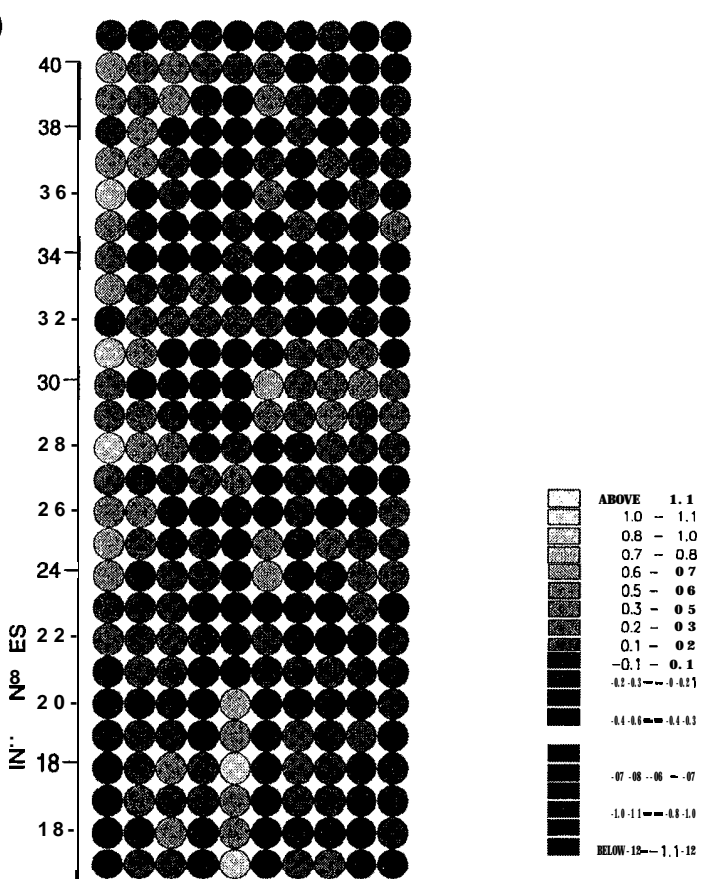

(2)

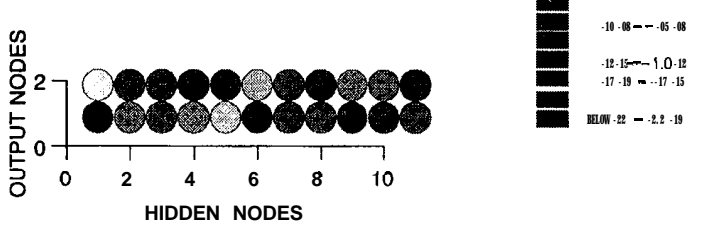

FIGURE 9. Weight map for a trained BPNN with 40 input nodes with notations as in Figure 8. This BPNN is trained with 10 pairs of $P$-arrival and background noise segments of vertical component recordings. 
